\title{
ANÁLISIS SEDIMENTOLÓGICO Y FACIOLÓGICO DE LA FORMACIÓN ROCHA (ex Grupo Rocha)
}

\author{
LEDA S. BETTUCCI *** \& ADRIANA M. BURGUEÑO ****
}

\begin{abstract}
SEDIMENTOLOGY AND FACIOLOGIC ANALYSIS OF ROCHA FORMATION (EX-ROCHA GROUP). The aim of this work is to contibute to the sedimentologic and faciologic knowledge of the so called Rocha Group. The metasedimentary sequence structures are described and characterized by textural and compositional analysis. The sequence presents low grade metamorphism and deformation in large areas. A more detailed study was done in areas where there is no conspicuous deformation, based on field evidence. The present distribution of the metasediments suggests a northwestward basal portion. From the results and interpretation of the facies units, Formación Rocha is proposed to include all the low grade supracrustals of the Rocha Group.
\end{abstract}

Keywords: Metamorphosed sediments, facies units, Rocha Group, Rocha Formation, Proterozoic.

\begin{abstract}
RESUMEN El propósito del presente trabajo es contribuir al conocimiento faciológico y sedimentológico del hasta ahora denominado Grupo Rocha. Se describen y caracterizan las estructuras de los paquetes metasedimentarios mediante un análisis textural y composicional de los mismos. Esta secuencia presenta un metamorfismo de grado bajo y con extensas áreas deformadas. El estudio detallado se efectuó tomando las áreas con mínima deformación, según criterios de campo. Tal como se presentan actualmente los metasedimentos, la base de depósito estaría ubicada hacia el NW. De los resultados e interpretaciones de las unidades faciológicas se propone definir la Formación Rocha para agrupar a las supracrustales de bajo grado integrantes del Grupo Rocha.
\end{abstract}

Palabras clave: Metasedimentos, unidad faciológica, Grupo Rocha, Formación Rocha, Proterozoico.

INTRODUCCIÓN El propósito del presente trabajo es contribuir al conocimiento sedimentológico y faciológico de la hoy denominada Formación Rocha.

Del relevamiento geológico a Escala 1:100.000 (Masquelin et al. 1988, Oyhantgabal et al 1993, Preciozzi et al. 1993) surge este trabajo; se describió y caracterizó la estructura de los paquetes metasedimentarios. Para tal efecto, se efectuó un análisis textural y composicional de los metamorfitos, y de las estructuras sedimentarias primarias preservadas en ellos.

Para esta secuencia proterozoica, metamorfizada y deformadas, se extrapolaron aquellas definiciones aplicables a sedimentos fanerozoicos. Sin embargo, no se deben olvidar las limitaciones que existen en el conocimiento de la duración de los ciclos, las variaciones relativas del nivel del mar, la tectónica y las limitaciones geocronológicas. La secuencia estudiada presenta un metamorfismo de grado bajo y con extensas áreas con mucha deformación, por esta razón el estudio detallado de la misma se efectuó donde la deformación es aparentemente mínima. Consecuentemente, los resultados fueron evaluados teniendo en cuenta las limitaciones de la extrapolación y asegurando la objetividad mediante la selección de secuencias representativas.

ANTECEDENTES El Grupo Rocha fue definido por Hasui et al. (1975). Posteriormente, Fragoso-Cesar et al. (1987) describen la presencia en las supracrustales del Grupo Rocha tres unidades litoestructurales (Unidad Cuarcítica, Unidad de Esquistos Verdes y Unidad Faro La Paloma), desarrolladas en la zona oriental del Cinturón Dom Feliciano, caracterizadas por una deformación y un metamorfismo decreciente hacia el este (Unidad Faro La Paloma). Según eses autores, la Unidad de Esquistos Verdes provendría de un posible protolito vulcanogénico básico y la Unidad Faro
La Paloma (playas La Paloma y La Pedrera) representaría el área con menos metamorfismo.

Los metamorfitos de esta unidad litoestratigráfica han sido considerados como parte del Grupo Lavalleja (Bossi et al. 1975) y del Grupo Lavalleja - Rocha (Preciozzi et al. 1979, 1985). Bossi \& Navarro (1988) proponen la denominación de Grupo Rocha para los metamorfitos de medio y bajo grado, de rumbo regional $\mathrm{NE}$, que se desarrollan del este de la ciudad de Rocha hasta algunos kilómetros al oeste del Balneario La Coronilla.

METODOLOGÍA El área de estudio se halla situada en el Departamento de Rocha, Uruguay (Fig. 1). La descripción detallada de la misma se encuentra en la tabla 1. Para las localidades donde aparece representado el Grupo Rocha, realizáronse un total de 27 cortes geológicos en siete fotoplanos, se describieron 281 afloramientos y se realizaron un total de 55 láminas delgadas, las cuales son representativas de las litologías revisadas.

A partir de las observaciones de campo se reconocieron metasedimentos, los que se separaron en una primera etapa en metapsamitas y metapelitas. Estas litologías aparecen con una determinada distribución en el área de estudio. Para su caracterización más detallada, seleccionáronse muestras a las que se les realizaron láminas delgadas y así se llegó a la composición mineralógica y a sus componentes texturales. De esta manera, fue posible corroborar la definición litológica efectuada a nivel de campo.

Como criterio de clasificación de las metapsamitas, se utilizó el diagrama de Pettijohn et al. (1972, modificado de Dott 1964).

Se reconstruyeron una serie de cortes geológicos con los que se realizaron ocho perfiles que comprendían a las zonas de mínima deformación. A partir de dichos perfiles, fue posible separar una serie de unidades litoestratigráficas.

\footnotetext{
* Dirección Nacional de Minería y Geología, DI.NA.MLGE, Casilla 11800, Hervidero 2861, Montevideo, Uruguay; y Facultad de Ciencias, Departamen-
} to de Geología, Casilla 11400, Tristán Narvaja 1674, Montevideo, Uruguay 


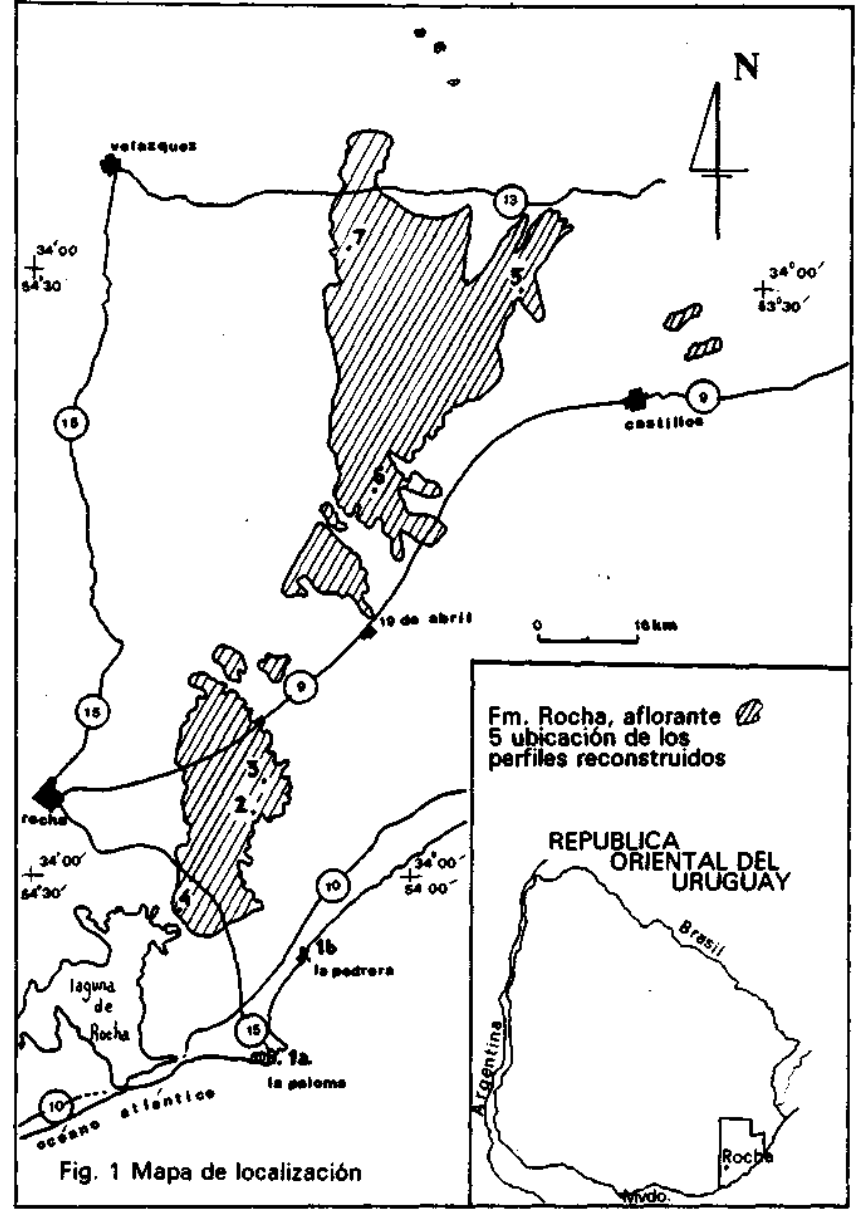

Figura 1-Mapa de localización

Figure 1 - Location map

RESULTADOS La Formación Rocha se caracterizó a partir de las descripciones realizadas en las áreas aflorantes, indicadas en la tabla 1 .

La secuencia metasedimentaria está representada por un conjunto de metapsamitas y metapelitas. La distribución granulométrica dentro de la Formación muestra el predominio de la fracción arena (Fig. 2). En la figura 3 se observa que dentro de la fracción arena la granulometría dominante es la arena fina. La figura 4 muestra que, dentro de la fracción limos, domina la fracción limos medios.

Tabla 1 -Área de estudio

Table - Study area

\begin{tabular}{l|c|c|c|c}
\hline Fotoplano & $\begin{array}{c}\text { Area aprox. } \\
\text { del grupo }\end{array}$ & $\begin{array}{c}\text { Corte } \\
\text { Geol. }\end{array}$ & $\begin{array}{c}\text { Aflor. } \\
\text { descr. }\end{array}$ & LD. \\
\hline Velazquez & $145 \mathrm{~km}^{2}$ & 11 & 118 & 12 \\
\hline $\begin{array}{l}\text { Rocha y La } \\
\text { Paloma }\end{array}$ & 185 " & 9 & 89 & 15 \\
\hline Chafalote & 204 “ & 3 & 34 & 11 \\
\hline Castillos & 184 " & 2 & 30 & 14 \\
\hline I. Muerta & $40 "$ & 1 & 8 & 2 \\
\hline A. Dulces & 3 "\# & 1 & 2 & 1 \\
\hline
\end{tabular}

\# No representa el área total de la Formación dentro de este Fotoplano. $\mathrm{LD}=$ Láminas delgadas

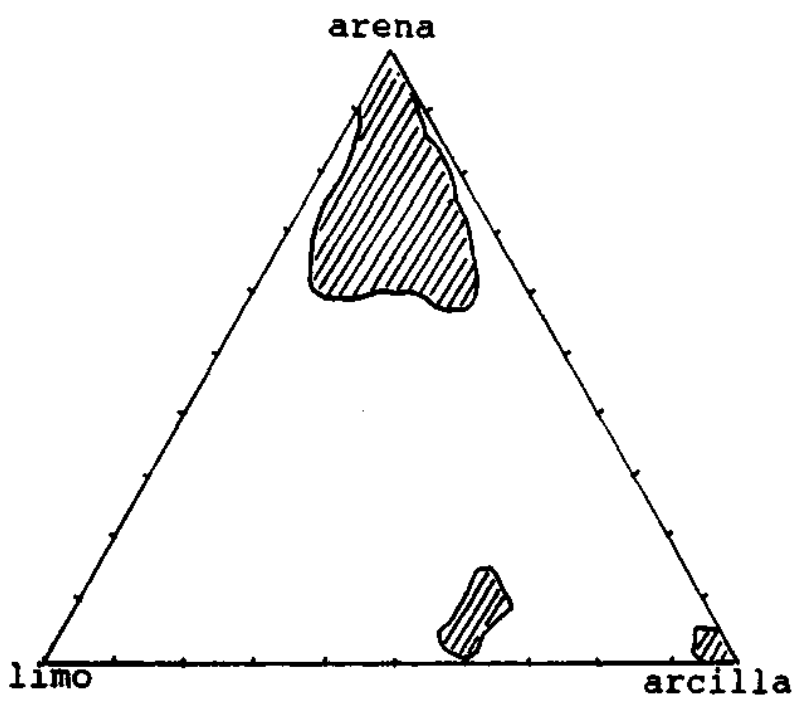

Figura 2-Dominios granulométricos Figure 2 - Grain size domains

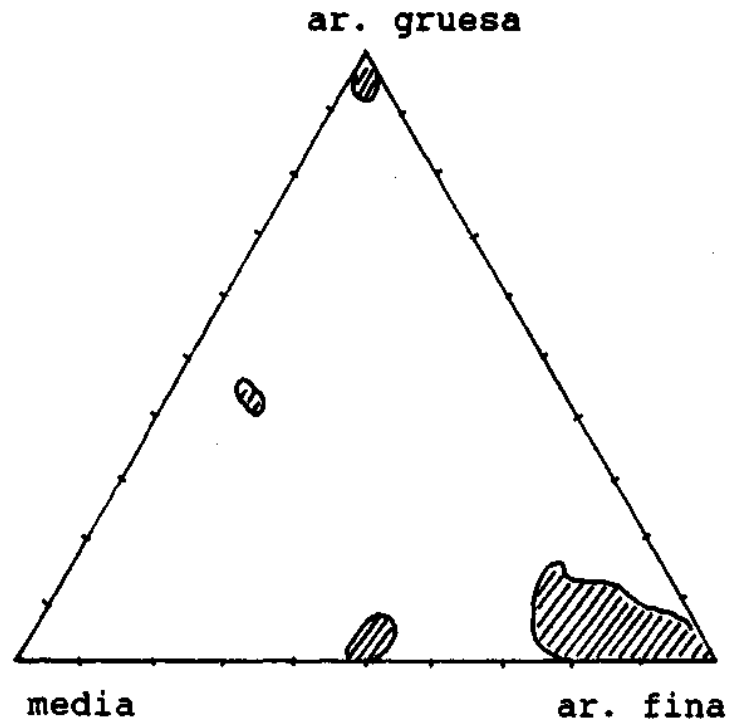

Figura 3-Dominios granulométricos de las arenas Figure 3 - Grain size domains for the sand fraction

Los diagramas de selección granulométrica se realizaron según la escala de Wentworth. En la figura 2 el extremo correspondiente a las arcillas no indica los minerales arcillosos sino la fracción granulométrica representada por filominerales, principalmente muscovita.

En los trabajos realizados en los fotoplanos mencionados (Tab. 1), no se encontraron términos efusivos.

El estudio detallado de las láminas delgadas mostró que la mineralogía más repetida es: $\mathbf{1}$. cuarzo + plagioclasa + muscovita + (clorita); 2. cuarzo + plagioclasa (oligoandesina) + muscovita + (biotita) + (turmalina) + clorita + opacos y como minerales accesorios: epidoto, esfeno, circón, apatito, calcita, pirita.

Para el caso de la biotita, esta ocurre principalmente en áreas sometidas a metamorfismo térmico producido por las intrusiones graníticas.

Descripción de Unidades Faciológicas Se han caracterizado tres unidades faciológicas. 
limo grueso

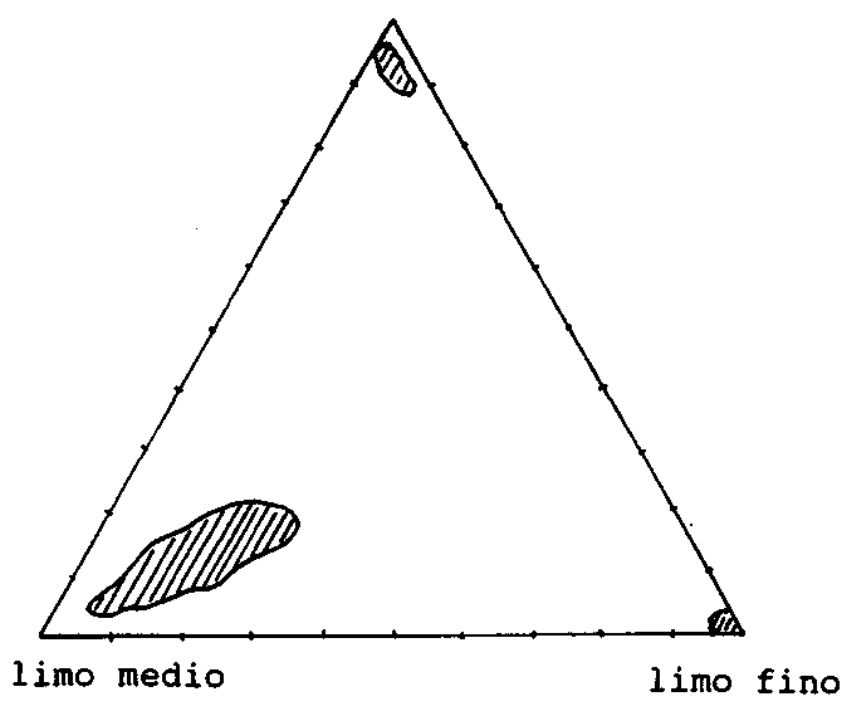

Figura 4 - Dominios granulométricos de los limos Figure 4 - Grain size of the silt fraction

1. Unidades pelíticas a. Facies de metapelitas masivas. Representadas por metasedimentos limo arcillosos. A partir de los datos de las láminas delgadas, se observó que no existen estructuras sedimentarias más que una laminación incipiente de origen metamórfico. En algunos casos, pre-

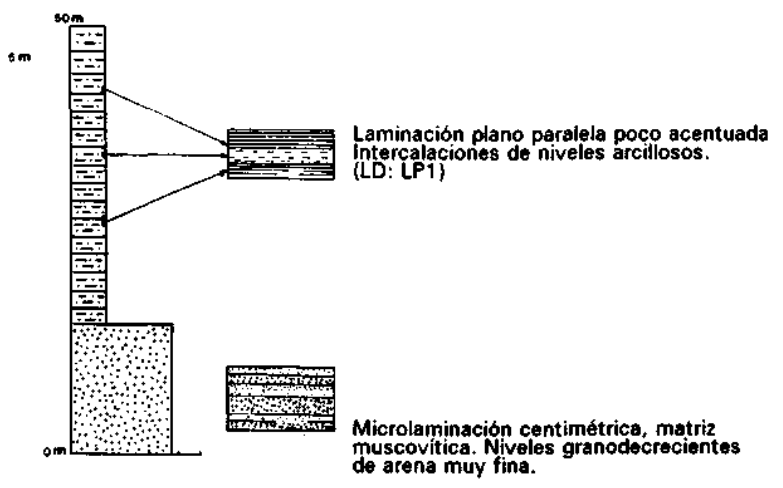

REFERENCIAS

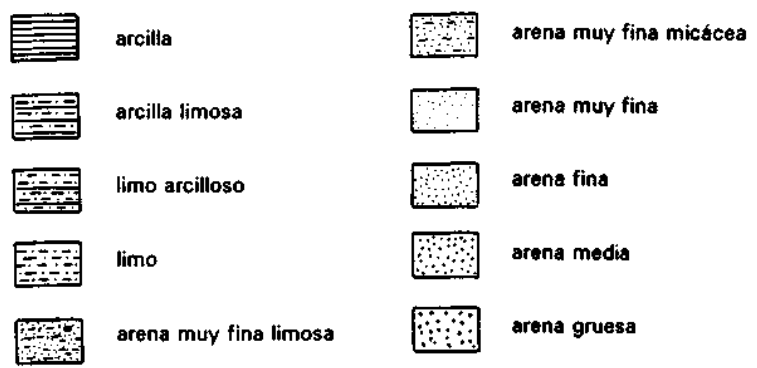

sentan granos dispersos de cuarzo y plagioclasa de tamaños medios a gruesos.

Figura 5 - Perfil la "Cabo Santa Maria"

Figure 5 - Cabo Santa María section

b. Facies de metapelitas laminadas. Representadas también por materiales de granulometría predominantemente limo$\mathrm{sa}$, con pequeñas alternancias de arcillas. Hacia el tope del Perfil 2 (Fig. 7), se encontró un paquete pelítico donde se constató la presencia de lentes de arena fina y canales granodecrecientes. Ambos en cuerpos arcillo-limoso con pasaje lateral a limos. En el caso del Perfil 3 (Fig. 8), se destacó la presencia de un paquete de granulometria arena, considerado como un pulso debido a su escasa potencia, donde se pudo identificar una interestratificación de arena muy fina y media. En este paquete las láminas delgadas confirman la presencia de estos materiales.

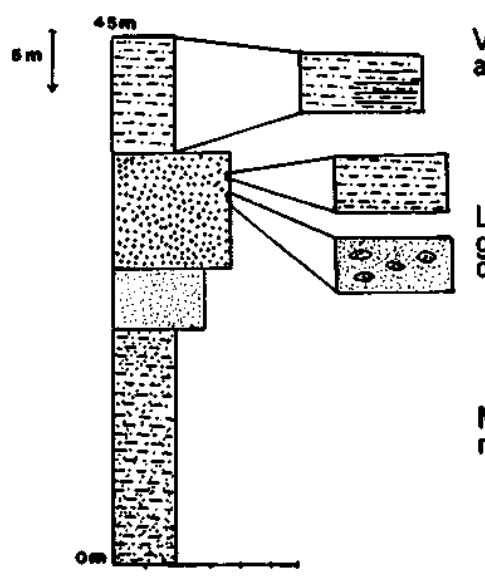

Variación lateral de limo a arcilla limosa (LD: LPr1)

Lentes de arena media a gruesa de hasta $10 \mathrm{~cm}$. en cuerpo de arena fina

Figura 6 - Perfil Ib "La Pedrera" Figure 6 - La Pedreira section $1 \mathrm{~b}$

2. Unidades metapsamíticas a. Facies de metapsamitas masivas. Este caso está representado por paquetes de metapsamitas medias.

b. Facies de metapsamitas granodecrecientes. Son potentes paquetes que se encuentran representados en los Perfiles 7 (Fig. 12) y 5 (Fig. 10), varían desde la fracción arena gruesa a fina. Ocurren también granodecreciencias centi a decimétricas de fracción arena media a arena fina. c. Facies de metapsamitas finas con estratificación planoparalela. Se determinó, a través de láminas delgadas, que la granulometría dominante era arena muy fina y fina. Las laminaciones están dadas por la presencia de materiales pelíticos. En los Perfiles 1b, la y 3 (Figs. 6, 5 y 8 respectivamente), se identificó la presencia de arena media en estos cuerpos. En los primeros dos perfiles antes mencionados se observó también, dentro de las metapsamitas finas, laminaciones centi a decimétricas granodecrecientes.

3. Unidades ritmicas metapsamitas-metapelitas Estas unidades rítmicas están dadas por la sedimentación alternada de materiales netamente pelíticos con materiales psamíticos y en otros casos materiales arcillosos y limosos.

Se describen a continuación las características generales de los afloramientos a partir de los cuales se llevó a cabo la reconstrucción de los Perfiles.

En lo que corresponde a los fotoplanos Rocha y La Paloma se reconstruyeron cinco perfiles donde se pueden observar una serie de estructuras. Para el caso del Perfil la (Fig. 5), destacase la presencia de niveles granodecrecientes y microlaminaciones de metapelitas. En el Perfil 1b (Fig. 6), se observa hacia el tope lentes de arena media a gruesa en un cuerpo de metapsamita fina. En el Perfil 2 (Fig. 7), se reconocieron estructuras de canal y lentes metapsamíticos en cuerpos metapelíticos. En el Perfil 3 (Fig. 8), se observó la predominancia de materiales metapelíticos donde aparece un cuerpo interestratificado de metaareniscas muy finas con metaareniscas medias. En el Perfil 4 (Fig. 9), los materiales eran predominantemente metapsamíticos. 


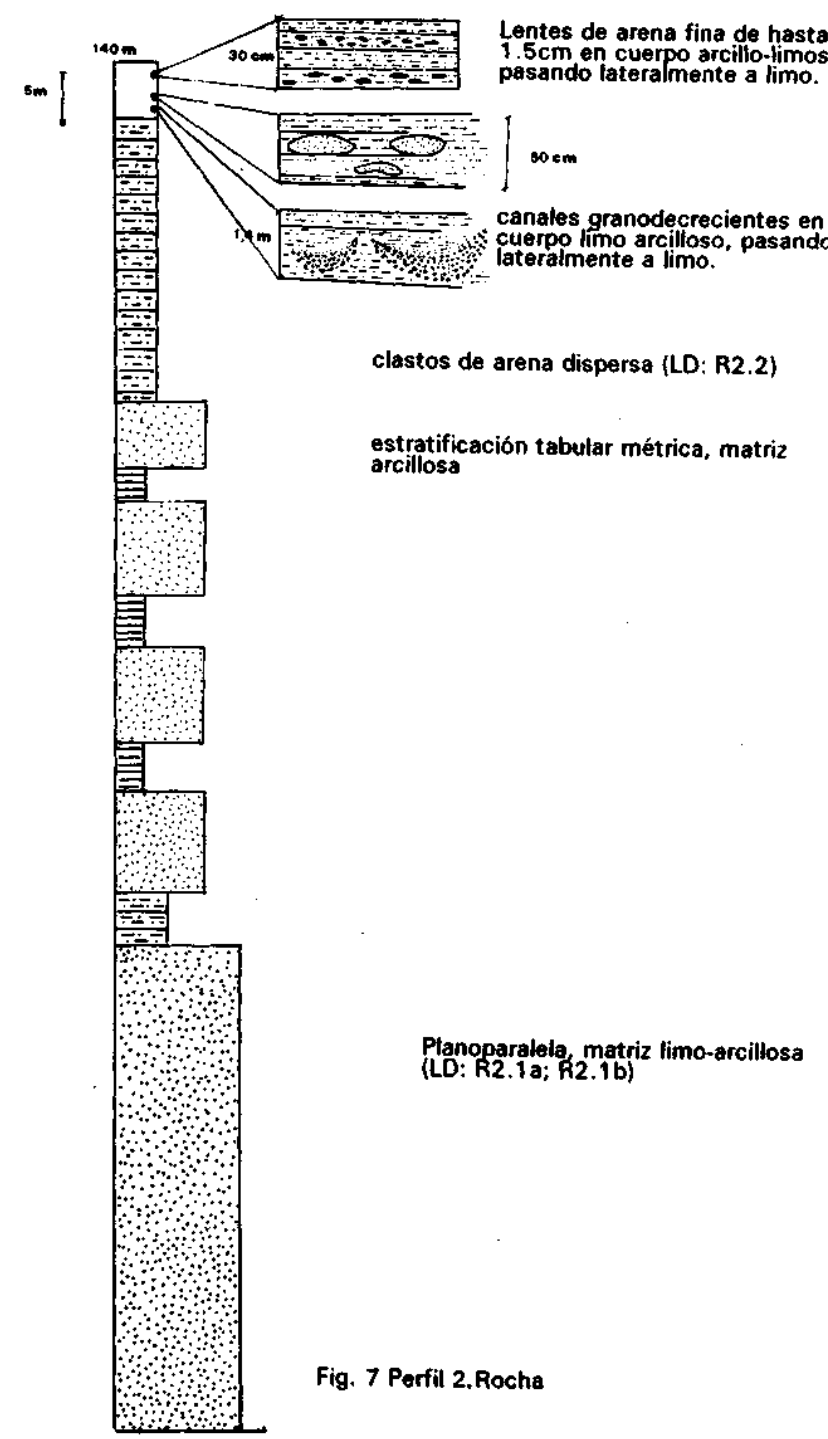

Figura 7 -Perfil 2 "Rocha"

Figure 7 - Rocha section 2

El Perfil 5 (Fig. 10), que se reconstruyó para el Fotoplano Castillos, se observó una clara alternancia de paquetes metapsamíticos con paquetes metapelíticos.

El Perfil 6 (Fig. 11), que se reconstruyó para el Fotoplano Chafalote, las litologías predominantes eran metapelíticas y algunas con microlaminación.

El Perfil 7 (Fig. 12), que se reconstruyó de la Formación Rocha comprendido en el Fotoplano Velázquez, muestra de base a tope la siguiente secuencia de paquetes: metapsamitas granodecreciente, metapelitas (limo arcillosas) con microlaminación interna, metapelitas (limo arenosas), metapelitas masivas, arcillo limosas.

El Perfil 8 (Fig. 13), que se reconstruyó para el fotoplano India Muerta, muestra principalmente la presencia de dos paquetes masivos, uno metapelítico hacia la base y otro metapsamítico hacia el tope.

En la región de Aguas Dulces, se realizó una única observación donde se describió una alternancia de paquetes métricos de metapelitas marrón claro y verdes, con paquetes de metapsamitas medias a finas. Las interpretaciones del mismo no fueron tomadas en cuenta debido a la alta deformación que presentaban.

De acuerdo a los relevamientos de campo realizados, no es posible separar unidades estratigráficas formales

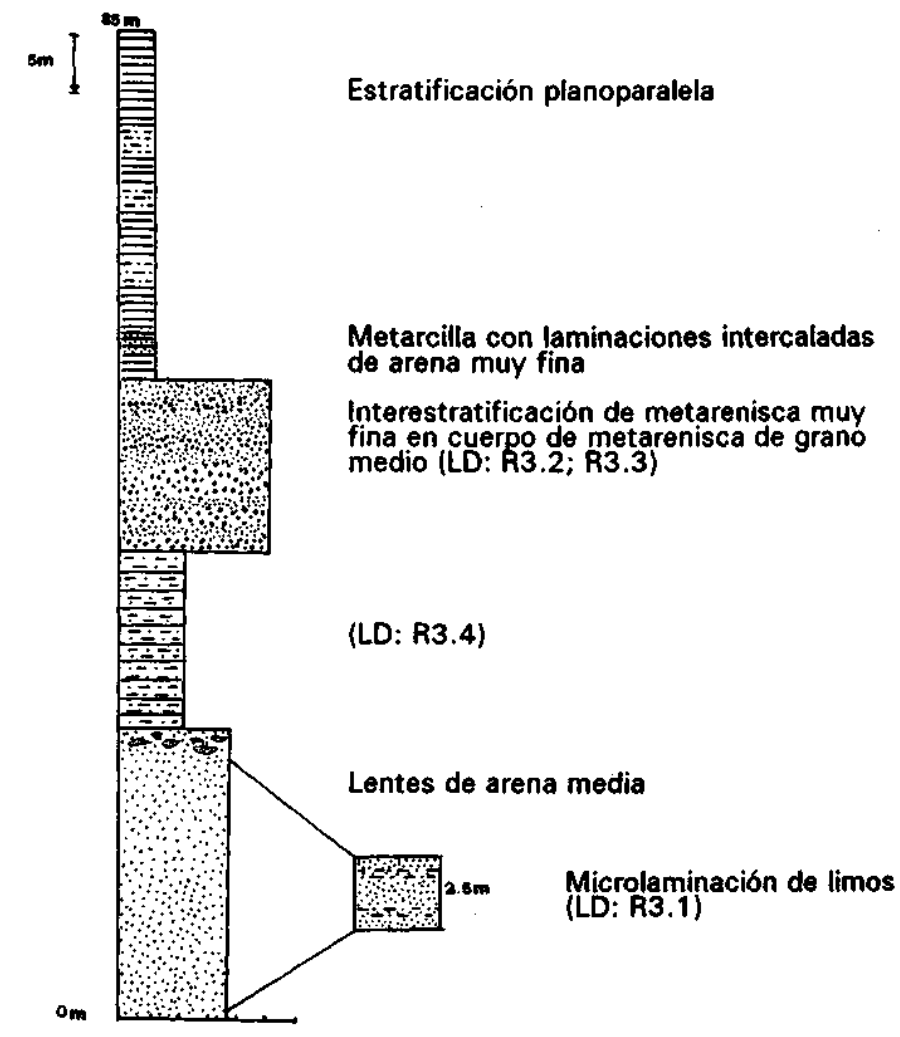

Figura 8 - Perfil 3 "Rocha"

Figure 8 - Rocha section 3

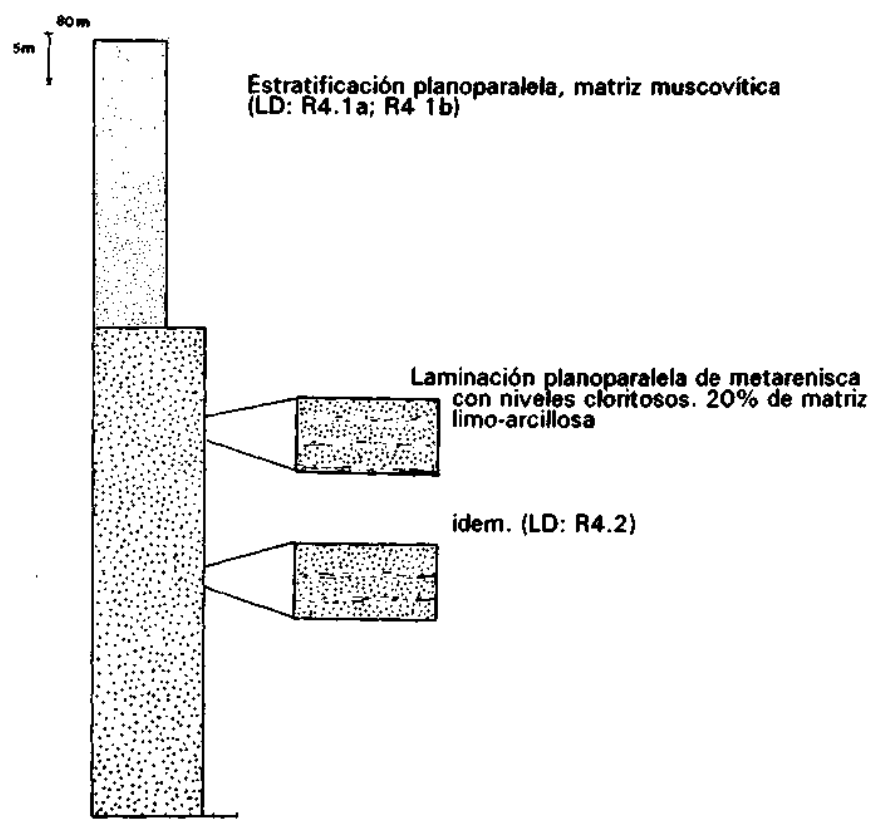

Figura 9 - Perfil 4 "Rocha"

Figure 9 - Rocha section 4

como indica el Código Estratigráfico. En general la relación grano-matriz resultó ser de moderada a alta.

Las estructuras sedimentarias preservadas que se observaron fueron: estratificación horizontal plano-paralela; estratificación lenticular, con lentes de hasta un centímetro de granulometrías finas. Aparecieron también lentes de hasta un metro de eje meyor, de materiales finos; intraclastos arcillosos y limo-arcillosos; microlaminación; canales macizos con pobre selección, grosera gradación textural, de grano grueso y borde neto; granodecrecencias de paquetes 


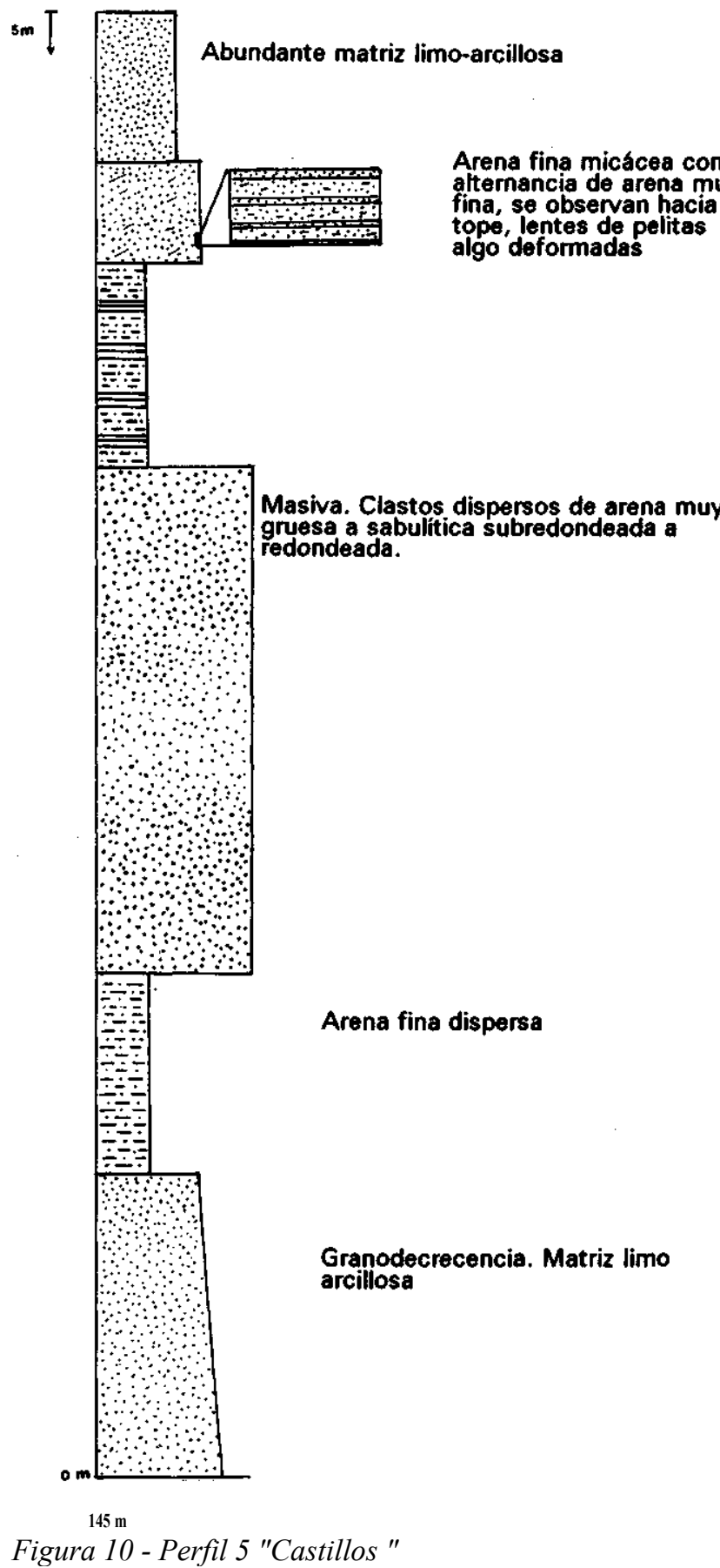

Figure 10 - Castillos sections

pequeños y menos frecuentemente en paquetes de mayor potencia; ritmicidad en paquetes metapsámiticos y metapelíticos.

Con respecto al carácter composicional, su estudio reveló la predominacia de cuarzo (Tab. 2). La plagioclasa es de tipo oligoandesina (en proporción menor al 10\%), apareciendo muy raras veces feldespato potásico, generalmente alterado. Los accesorios (menor al 1\%) correspondieron, generalmente a turmalina, epídoto, circón, apatito, y en los filominerales se destacaron sericita, muscovita y clorita.

Texturalmente dominan los granos subangulosos a subredondeados, principalmente para la fracciones arena muy fina a media. De las características mineralógicas y texturales de las muestras analizadas se infiere una madurez media.

DISCUSIÓN Los resultados de este estudio sugieren una continuidad litológica desde la playa La Paloma a la

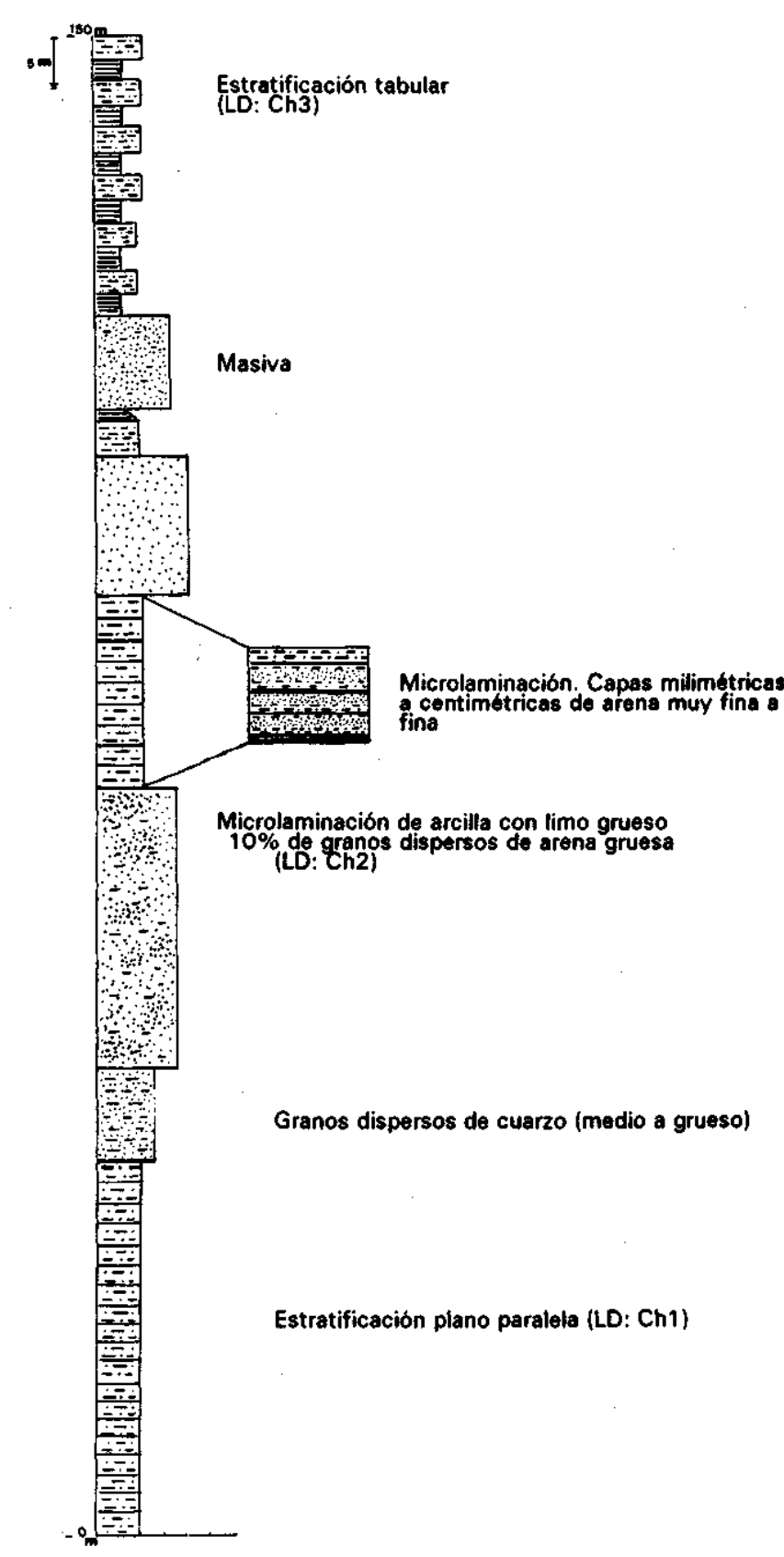

Figura 11-Perfil 6 "Chafalote"

Figure 11 - Chafalote section 6

porción más occidental de la Formación Rocha. Por lo que no es posible separar la Unidad Faro La Paloma (FragosoCesar et al. 1987) del resto de las unidades integrantes de la formación. Las observaciones geológicas muestran que se trata de un solo conjunto metasedimentario.

Fragoso-Cesar et al. (1987) señala la existencia de esquistos cloritosos a los que les atribuye un carácter vulcanogénico básico, asimilando cristales de cuarzo redondeado a primitivas vacuolas de la lava original. No obstante, en el trabajo no fue posible verificar la existencia de un vulcanismo básico, en cambio se han encontrado metasedimentos cloritosos con un claro origen sedimentario.

Los materiales metasedimentarios que integran la hoy definida Formación Rocha son silicoclásticos, constituidos por metaareniscas wackicas a subwackicas y metapelitas. Las unidades metasedimentarias preservadas muestran contactos netos, siendo la base y el techo planares. 
Tabla 2 - Descripción de láminas delgadas

Tabela 2 - Thin section description

\begin{tabular}{|c|c|c|c|c|c|c|c|}
\hline \multicolumn{2}{|c|}{ No. LD Fotoplano } & \multirow{2}{*}{$\frac{\begin{array}{l}\text { Granu- } \\
\text { lometria }\end{array}}{\text { Arc-limosa }}$} & \multirow{2}{*}{ Textura } & \multirow{2}{*}{ Comp. mineralogica } & \multirow[t]{2}{*}{$\% \mathrm{mtz}}$. & \multirow{2}{*}{ Litología } & \multirow{2}{*}{$\begin{array}{l}\text { Observaciones } \\
\text { Frace. limo: qz, pg. fm: musc, (cl). }\end{array}$} \\
\hline LP1 & La Paloma & & & & & & \\
\hline LP2 & La Paloma & Ar. f a $m$ & SA - SR & $q z, p g, f m$ & 15 & metarenisaca subwackica & mtz: musc y cl; limo m.: qz \\
\hline LPR1 & La Paloma & Arc-limosa & & fm & & metapelita & qz y pg en fracc. ar. gr. \\
\hline LPR2 & La Paloma & Ar. $\mathrm{mf}$. & $\mathbf{S R}-\mathbf{R}$ & $q z, p g$, tur, ox de Fe & 25 & metarenisca wackica & mtz: muse, ser, cl, (bt) \\
\hline $\mathbf{R} 2.2$ & Rocha & limo gr. & $\mathbf{S R}$ & qz, pg, (tur, ep, zr) & 10 & metapelita muscovítica & mtz: musc-ser \\
\hline R2.1a & Rocha & Ar. $\mathbf{m}$ & SR & $q z, p g$, tur, fm. & 40 & metarenisca calcárea & mtz: calc; pg algo alterada \\
\hline R2.1b & Rocha & Ar. $m$ & SR & $q z, p g$, ep, op, musc, cl & 20 & metarenisca wackica & mtz: musc, (cl) \\
\hline R3.1 & Rocha & Ar. mf. af. & SR - SA & qz. pg. (op, musc, cl) & 30 & metarenisca wackjea & mtz: limo-arc (musc, bt, cl) \\
\hline $\mathrm{R} 3.2$ & Rocha & Ar. $\mathrm{m}$ & $\mathbf{S R}$ & qz, pg, tur, zr, op, musc & 20 & metarenisca wackica & mtz: limo-arc. \\
\hline R3.3 & Rocha & Ar. mf̣. & SA - SR & $q z, p g,(e p, f m)$ & 10 & metarenisca & mtz: musc, cl; granos de ar. m. dispersa \\
\hline $\mathrm{R} 3.4$ & Rocha & limo & SA & $\mathrm{qz}, \mathrm{pg}$, musc & & metapelita & ar. f. dispersa \\
\hline R4.1a & Rocha & Ar. $\mathrm{mf}$ a $\mathrm{f}$ & SA - SR & $q z, p g, f m, e p$ & 15 & metarenisca subwackica & mtz: musc \\
\hline $\mathrm{R} 4.1 \mathrm{~b}$ & Rocha & At. $\mathrm{mf}$. & SA - SR & $q z, p g, f m$ & 10 & metarenisca & mtz: musc, $c l, q z$ y pg subautomorfa ( $g x$ ) \\
\hline R4.2 & Rocha & Ar. f. & SR - SA & $q z, p g, f m$, ap, $2 r$, ep & 15 & metarenisca subwackica & mtz: musc, cl, bt, qz \\
\hline $\mathrm{CH} 1$ & Chafalote & limo-arc & & qz, musc, (cl) & & metapelita & \\
\hline $\mathrm{CH} 2$ & Chafalote & Ar. $\mathrm{mf}$ & SA & qz. pg, musc, cl & 20 & metarenisca wackica & \\
\hline $\mathrm{CH3}$ & Chafalote & Arc-limosa & & $\mathrm{fm}, \mathrm{qz}$ & & metapelita & clastos de $\mathrm{gz}$ y $\mathrm{pg}$ fracc. limo $\mathrm{m}$. \\
\hline $\mathbf{M} \mathbf{r}$ & India Muerta & limo-arc & & $\mathrm{fm}, \mathrm{qz}, \mathrm{pg}$ & & metapelita & \\
\hline IM2 & India Muerta & Ar. $\mathrm{m}$. & SA - SR & $q z, p g,(e p), f m$ & 15 & metarenisca & mtz: musc, (cl) \\
\hline $\mathrm{Cl}$ & Castillos & Ar. $\mathrm{mf}$ & SA - SR & $q z, p g, o p$ & 10 & metarenisca polimfcltica & clastos de ar. gr. đispersa \\
\hline
\end{tabular}

Ar: arena, gr: gruesa, m: media, f: fina, mf: muy fina, are: arcilla; qz: cuarzo, pg: plagioclasa, fm: fílominerales, ep: epidoto, op: opacos, el: clorita, muse: muscovita, bt: biotita, tur: turmalina, zr: circón, ap: apatito, ser: sericita.

mtz: matriz, R: redondeado, SR: subredondeado, SA: subanguloso

Hacia el tope de algunos cuerpos metapsamíticos se han observado estructuras ondulíticas.

La preservación de estructuras sedimentarias sugiere que el grado de deformación de estos materiales es bajo, como así también las paragénesis observadas indican un grado de metamorfismo bajo.

Los materiales hasta ahora descriptos indican eventos turbidíticos en los cuales aún no se han separado secuencias de Bouma.

De acuerdo a la geometría de los cuerpos que es tabular, la continuidad, las estructuras preservadas y las características composicionales y texturales, estaríamos frente a una cuenca marginal marino profundo no descartándose la posibilidad de una situación plataformal.

En función de la disposición actual de los metasedimentos se observa que existe un paulatino decrecimiento granulométrico hacia el SE. Esto se refleja en los potentes paquetes de metapelitas hacia el SE que se alternan con paquetes metapsamíticos menos potentes. A su vez se encuentran cuerpos cuarcíticos hacia el NW desapareciendo hacia el SE. Las estructuras de canales encontradas en los paquetes metasedimentarios del Fotoplano Rocha, ubicadas en la zona central de esta Formación y las granodecrecencias centi a decimétricas que se encuentran en la zona más oriental son otro argumento para creer que la base de ésta se encuentra hacia el NW.

Los resultados e interpretación de las unidades faciológicas que presentan continuidad litológica hacen altamente aconsejable que los metasedimentos de grado bajo, pertenecientes al Grupo Rocha pasen a integrar una Formación, Formación Rocha. Cabe destacar que el nombre de esta formación coincide con el nombre del Grupo, debido a que estos metamorfitos de grado bajo se presentan en distintas localidades del departamento de Rocha, antes mencionadas, y no se describe un perfil tipo en la Ciudad de Rocha, exclusivamente. Las autoras del presente trabajo sugieren denominar al resto de las litologías incluidas por Bossi \& Navarro (1988), tales como los metamorfitos de grado medio ubicados en la región más occidental, como Grupo Aiguá-Paralle, hasta 

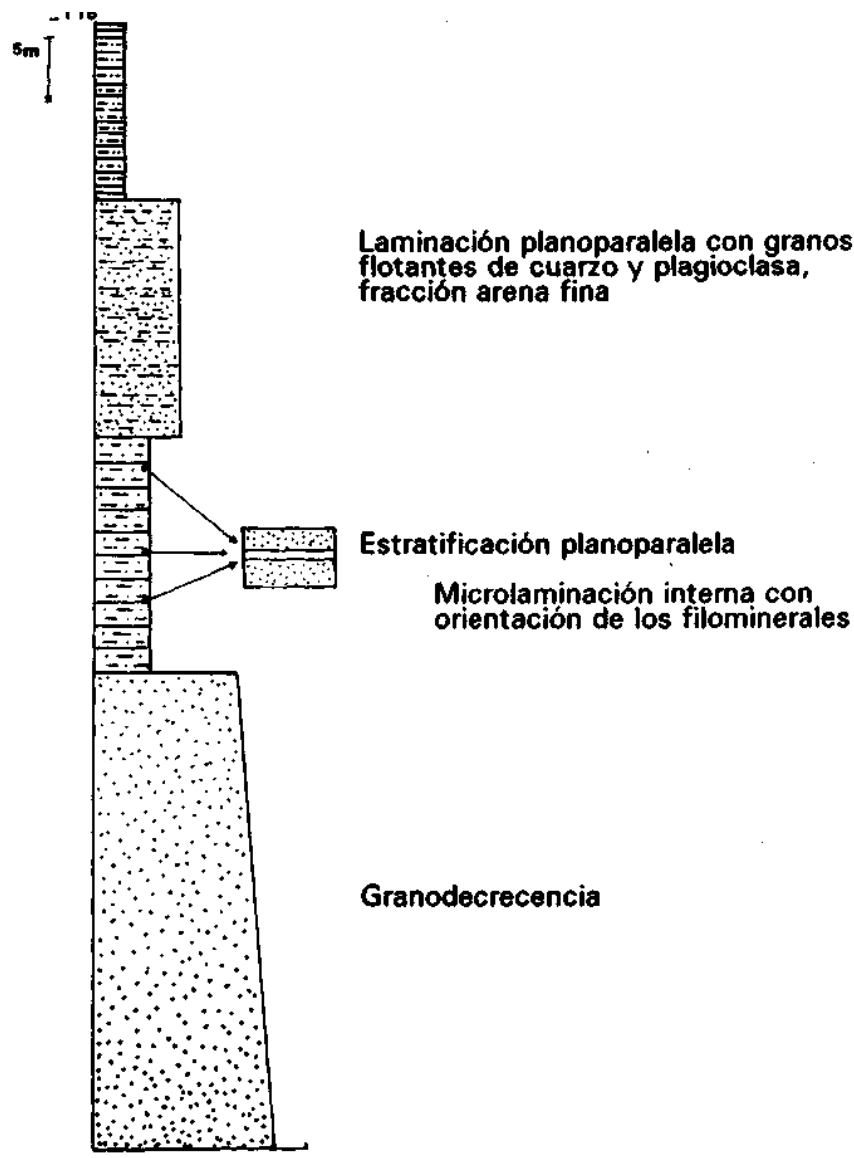
fotantes de cuaro y plagioclasa, racción arena fina

Estratificación planoparalela

Microlaminación interna con orientación de los filominerales

Granodecrecencia

Masiva

Figura 12 - Perfil 7 "Velazquez"

Figure 12 - Velazquez section 7

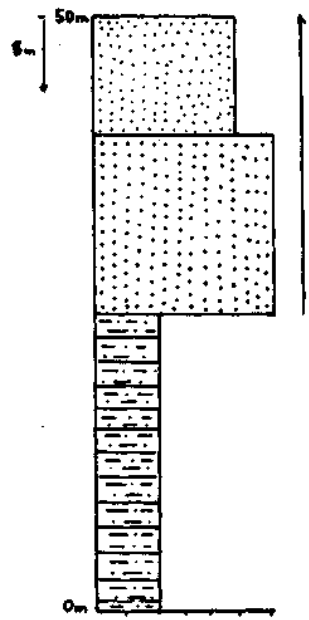

Masiva (LD: IM2)

Incremento de muscovita en la matriz

Masivo (LD: IM1)

Figura 13 - Perfil 8 "India Muerta" Figure 13 - India Muerta section 8

una mejor revisión de las litologías que hasta hoy integran el Grupo Rocha.

Agradecimientos Deseamos agradecer al Sr. A. Sandes por su gran esmero en la realización de las láminas delgadas; a los técnicos de la DLNA.MLGE quienes compartieron las tareas de campo. Agradecemos especialmente al Dr. Fernando Preciozzi, Director de la División Geología Básica (DLNA.MLGE.) y a los Licenciados Héctor de Santa Ana y Gerardo Verovslasky del Departamento de Geología de la Facultad de Ciencias por su confianza brindada. Igualmente a los revisores científicos de esta Revista.

\section{REFERENCIAS BIBLIOGRÁFICAS}

BOSSI, J:FERRANDO, L. FERNANDEZ, A.; ELIZALDE, G ; MORALES, H.; LEDESMA, J.J.; CARBALLO, E.; MEDINA, E.; FORD, L; MONTANA, J. 1975. Carta Geológica del Uruguay (1:1.000.000). Montevideo, Dir. Suelos y Fértil. Min. Agr. y Pesca.

BOSSI, J. \& NAVARRO, R. 1988. Geología del Uruguay. Montevideo, Universidad de la República. (Tomo I).

COMISSÁO AMERICANA DE NOMENCLATURA ESTRATIGRÁFICA 1978. Código de Nomenclatura Estratigráfica. São Leopoldo, Publicações DAGEO, UNISINOS.

FRAGOSO-CESAR, A.R.S.; WERNICK, E.; SOLIANI Jr., E. 1982. Associacões petrotectônicas do Cinturão Dom Feliciano (SE da plataforma Sul-Americana). In: CONGR. BRAS. GEOL., 32. Salvador, 1982. Anais... Salvador, SBG. v. 1, p. 1-12.

FRAGOSO-CESÄ, A.R.S.; MACHADO, R.; GÓMEZ RIFAS, C. 1987. Observacões sobre o Cinturão Dom Feliciano no escudo uruguaio e correlacões com o Escudo do Rio Grande do Sul. In: SIMP. SUL-BRAS. GEOL., 3. Curitiba, 1987. Atas... Curitiba, SBG. v. 2, p. 791-809.

HASUI, Y; CARNEIRO, C.D.R.; COIMERA, A.M. 1975. The Ribeira fold belt. Rev. Eras. Geoc. 5(4):257-266.

MASQUELIN, H. \& TABO, F. 1988. Memoria Explicativa de la Carta Geológica del Uruguay, Hoja Chafalote, Escala 1:100.000. Montevideo, DI.NA.MI.GE/Fac. de Agron. Fac. de H. y Ciencias.
OHYANTCABAL, R; SANCHEZ, L.: MASQUELIN, H.: MEDINA, I. 1993. Memoria Explicativa de la Carta Geológica del Uruguay, Hoja Velazquez, escala 1:100.000. Montevideo, DI.NA.MI.GE/Fac. de Agron.- Fac. de Ciencias. (Inédito).

PRECIOZZI, R; SPOTURNO, J.: HEINZEN, W. 1979. Carta Geoestructural del Uruguay, Escala 1:2.000.000. Montevideo, Inst. Geol. Ing. E. Terra.

PRECIOZZI, F.; SPOTURNO, J.; HEINZEN, W.; ROSSI, P. 1985. Carta Geológica del Uruguay, Escala 1:500.000. Montevideo, DI.NA.MI.GE/Ministerio de Industria, Energía y Minería.

PRECIOZ7I F. PENA S - SANCHEZ, L 1993a Memoria Explicativa de la Carta Geológica del Uruguay, Hojas Rocha y La Paloma, Escala 1:100.000. Montevideo, DI.NA.MI.GE/Ministerio de Industria, Energía y Minería. (Inédito).

PRECIOZZI, F. PENA, S.: SANCHEZ, L. 1993b Memoria Explicativa de la Carta Geológica del Uruguav. Hoja Castillos, Escala 1:100.000. Montevideo, DI.NA.MI.GE/Ministerio de Industria, Energía y Minería. (Inédito).

MANUSCRITO A776

Recebido em 31 de agosto de 1993 Revisão do autor em 25 de fevereiro 1994 Revisão aceita em 25 de fevereiro de 1994 